\title{
Scénarios pour les territoires MRC du Québec
}

\author{
Isabel Brochu \\ Université du Québec à Chicoutimi
}

\section{Introduction}

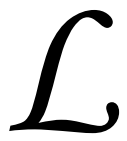
es territoires MRC (Municipalités régionales de comté) ont beaucoup progressé depuis leur création en 1979. Des enquêtes au fil du temps ont permis de démontrer que ce palier situé entre les municipalités locales et les régions administratives fut l'objet d'une réelle construction institutionnelle. En effet, diverses organisations publiques et collectives épousent désormais ces territoires dans leur gestion de fonctions publiques reliées à des biens et services collectifs desservis à la population
Les territoires MRC, ce palier situé entre les municipalités locales et les régions administratives, font l'objet d'une réelle construction institutionnelle.
Rouyn-Noranda, Trois-Rivières et Lévis modifient la dynamique des territoires MRC. Pour les principales agglomérations urbaines du Québec, leur proximité physique avec des territoires MRC et leur complémentarité opérationnelle dans l'exercice des compétences (aménagement, gestion de biens et services, soutien au développement) impliquent nécessairement une collaboration continue, ne serait-ce que pour concilier les usages du sol entre les vocations urbaine et rurale souvent en forte compétition. En 2003, le gouvernement du Québec a confirmé officiellement aux MRC et aux agglomérations urbaines la respon(aménagement, services sociaux et communautaires, matières résiduelles, sports, loisirs, évaluation foncière...), aux travailleurs (aides aux entreprises, recherche d'emploi, formation...) et aux entreprises (forêt, agriculture, tourisme, développement, financement...). Par l'entremise d'un judicieux mélange d'impulsions provenant du milieu (appropriation) et des instances supérieures (politique gouvernementale), les territoires MRC s'avèrent désormais institutionnellement occupés par diverses organisations. Ce processus ne semble pas terminé.

Récemment encore, certains efforts de réforme gouvernementale ont eu des incidences sur l'organisation territoriale de ces MRC. De 96 entités qu'elles étaient, il y a maintenant 88 MRC chapeautées par un Conseil de maires des municipalités locales. Notons que 10 de celles-ci sont localisées sur le territoire de la communauté urbaine de Montréal, alors que plusieurs gravitent autour de la ville de Québec, Gatineau et Sherbrooke. Soulignons aussi que 14 entités MRC épousent le statut de ville tout en exerçant des compétences de MRC.

En réalité, la réforme de l'an 2000 et l'apparition des grandes agglomérations urbaines comme Saguenay, sabilité en matière du développement local en confiant celle-ci directement au Conseil de ces entités. En même temps, le territoire régional a subi aussi une réorganisation, notamment avec la mise en place des Conférences régionales des élus (CRÉ). Ces éléments nouveaux, qui s'inscrivent dans le contexte d'une réforme territoriale progressive depuis près de quarante ans, risquent d'influencer considérablement la dynamique entre les divers territoires municipaux, MRC et régionaux.

Dans le cadre de cette grande réorganisation des territoires institutionnalisés qui composent le Québec, les organisations nationales telles l'UMQ (Uunion des municipalités du Québec), la FQM (Fédération québécoise des municipalités) et Solidarité rurale adoptent des positions très précises. Celles-ci influencent inévitablement les choix politiques concernant toutes les échelles territoriales. Pour l'UMQ actuellement, le renforcement des territoires et son corollaire, la décentralisation, concernent d'abord les municipalités locales qui représentent le seul palier politiquement imputable. Selon leur position, les municipalités doivent demeurer l'unique palier politiquement imputable. Pour la FQM et Solidarité rurale, le territoire MRC représente le palier de gouvernance le plus adé- 
quat pour accueillir de nouvelles compétences. Ces deux organisations revendiquent notamment le préfet élu au suffrage pour l'ensemble des MRC du Québec.

Ces positions tout à fait opposées jouent inévitablement un rôle important dans la progression (et la stagnation) de la réforme territoriale en cours, notamment sous l'angle du renforcement très lent des territoires MRC en quête de responsabilités publiques. L'exemple de la composante à l'égard des préfets élus illustre bien ce blocage institutionnel dont l'impact est important bien que non mesurable en matière notamment d'appropriation territoriale de facteurs de développement. Dans une perspective d'avenir, nous pouvons cependant saisir la situation institutionnelle actuelle à cet échelon MRC et analyser différents scénarios qui s'offrent dans le contexte sociopolitique plus large. Il s'agit de l'exercice que nous proposons dans cet article.

\section{I n'y a pas de règles établies en ce qui concerne la participation de la société civile organisée (association, organisme communautaire, groupe d'intérêt, etc.) et de la population dans l'exercice des fonctions du territoire MRC.}

\section{Les composantes de notre analyse}

Les territoires MRC illustrent une très grande diversité. Pour la saisir convenablement, il existe malheureusement peu de modèles pour analyser les divers attributs. Les apports de Fortin et Parent ${ }^{1}$, de MarcUrbain Proulx ${ }^{2}$ et de Jean Proulx ${ }^{3}$ ont cependant permis de faire des constats importants en regard de la construction de ces territoires et offrent un cadre pertinent pour évaluer les scénarios d'avenir.

Dans leur enquête, Parent et Fortin ont mesuré l'attitude divergente des décideurs lors de la mise en place des MRC sous l'angle de la participation de la population au processus décisionnel lors de l'implantation des Conseils MRC. Cette étude leur a permis notamment de dresser le profil de sept types de MRC allant de technocratique à participationniste. Nous croyons que les élus qui siègent aux Conseils MRC font face au même choix en ce qui concerne leur avenir. Nous retenons donc ces deux axes d'analyse, soit la technocratie et la participation, qui nous permettent de mettre en évidence les scénarios sous l'an- gle de la démocratie participative. Nous définissions ici l'approche technocratique comme étant celle qui consiste à faire appel uniquement à des experts, que ce soit à l'externe ou à l'interne, dans l'exercice des compétences, qu'elles soient anciennes, nouvelles, obligatoires ou facultatives. L'axe de la participation consiste donc à mesurer le degré d'ouverture sur le milieu dans l'exercice des fonctions. La consultation d’organismes, la création de comités, la consultation populaire et même l'élection du préfet sont autant de variables qui permettent de mesurer le degré de participation.

Les enquêtes de Proulx ${ }^{4}$ et Jean $^{5}$ visaient à mesurer le dynamisme des MRC québécoises. Elles ont mis en évidence que le dynamisme est une combinaison de la volonté des intervenants des territoires (pulsion endogène) et des multiples interventions de l'État (lois, réglementations, décrets, réformes, etc.). Nous retenons deux composantes de ces travaux, soit le dynamisme et l'appropriation. Le dynamisme se définit comme étant à la fois le nombre de fonctions exercées sur le territoire (santé, éducation, culture, développement, etc.) et l'approche utilisée par l'organisme qui exerce cette fonction. Par exemple, le développement local est une nouvelle fonction qui augmente l'indice de dynamisme du territoire. Cependant, le type d'approche utilisée par l'organisme peut augmenter ce dynamisme en facilitant la participation d'une diversité de décideurs et d'organismes. Ce qui nous amène à définir l'axe d'appropriation qui vise à évaluer les moyens adoptés par le Conseil MRC, ou les organismes du milieu, pour maximiser l'utilisation et l'adaptation des leviers disponibles au bénéfice du territoire MRC.

Pour mettre en évidence les scénarios qui s’offrent aux MRC, nous utiliserons quelques variables. La première est l'exercice des compétences obligatoires et facultatives puisque celles-ci sont au cœur même de l'existence des divers paliers de gouvernance et aussi du débat sur la décentralisation. Elle apparaît comme une variable essentielle à la réflexion sur les scénarios d'avenir. Le développement local, par l'entremise des centres locaux de développement, est désormais une compétence des MRC. Cependant, la formule utilisée peut faire l'objet de diverses applications, qui, selon nous, sont susceptibles de générer des résultats différents. Le CLD (Centre local de développement) est donc notre seconde variable. Finalement, les Conseils MRC disposent d'un nouvel outil, soit l'élection du 
préfet au suffrage universel. Malgré la faible demande de la part du milieu, il n'en demeure pas moins que le choix d'utiliser ou non cette option génère des modèles différents pour les MRC. Il en est de même pour le choix du préfet à temps plein. Nous avons donc retenu ces deux autres variables, préfet élu et à temps plein, dans l'analyse des scénarios d'avenir. Ces quatre grandes variables (compétences, CLD, préfet élu et préfet à temps plein) couvrent à la fois les responsabilités (anciennes et nouvelles) et les nouveaux outils dont disposent les MRC. Pour mettre en évidence ces variables, nous les examinerons à partir des deux grands composantes retenues pour notre analyse, soit la démocratie participative, sous les axes de la technocratie et de la participation, et le dynamisme des MRC, sous les axes du dynamisme et de l'appropriation.

\section{La démocratie participative}

Il n’y a pas de règles établies en ce qui concerne la participation de la société civile organisée (association, organisme communautaire, groupe d'intérêt, etc.) et de la population dans l'exercice des fonctions du territoire MRC. Le conseil de la MRC, composé des élus locaux, n'a pas d'obligation réelle d'ouverture sur le milieu. La consultation et la mise en place de comités, forums, tables sectorielles, groupes de réflexion, etc. sont laissées à leur entière discrétion. Les élus peuvent choisir une option qui favorise, de façon ponctuelle ou permanente, l'utilisation d'experts ou encore la mise en place de processus qui permettent une plus grande participation publique.

Le schéma 1 propose d'opposer les deux composantes que sont la technocratie et la participation en regard de trois variables : préfet temps plein (PTP), préfet élu (PÉ) et développement local (CLD). Si nous voulions illustrer tous les scénarios qui s'offrent aux MRC pour chacune de ces variables, les quadrants seraient composés d'une multitude de points dispersés puisque les possibilités sont grandes. Par exemple, un préfet élu peut travailler avec quelques organismes de façon ponctuelle, ou encore à quelques occasions dans une année ou de façon continue par des processus de consultation permanente. On constate qu'il est impossible de présenter chacune des possibilités, l’objectif est donc de s'attarder aux grandes tendances.

\section{Schéma 1 - Démocratie participative}

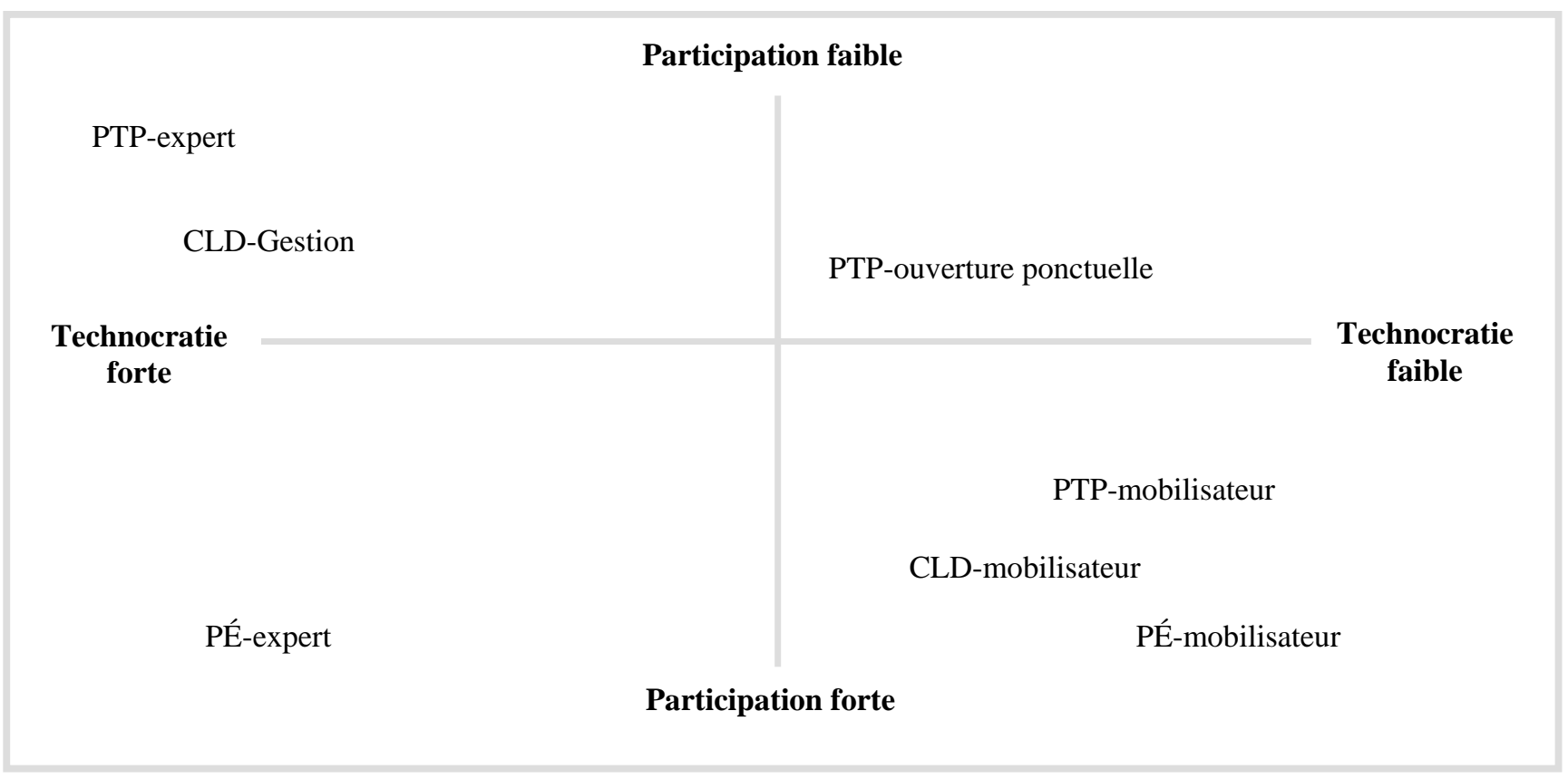

Les Conseils MRC ont la possibilité d'embaucher un préfet à temps plein sans l'élire. L'avantage potentiel est d'augmenter la disponibilité et permettre ainsi de s'occuper de dossiers plus complexes et même de donner plus de temps à des dossiers régionaux. Nous croyons cependant que la présence d'un préfet à 
temps plein ne signifie pas, de fait, une augmentation de la participation du milieu. Si le préfet à temps plein utilise uniquement des experts ou des ressources à l'interne sans mobilisation du milieu (PTP-expert), la MRC développera une approche plus technocratique avec une faible participation publique. À l'extrême, le préfet à temps plein peut adopter une vision mobilisatrice (PTP-mobilisateur) en créant divers mécanismes permanents de consultation et de concertation. Nous aurons une MRC de type participationniste. Entre les deux, il y a la possibilité de mobilisation ponctuelle ou selon certaines fonctions précises (PTP-ouverture ponctuelle).

Le préfet élu relève de la démocratie représentative. Cependant, une enquête effectuée en 2005 auprès des sept MRC ayant un préfet élu nous permet d'avancer que l'élection au suffrage universel favorise une participation du public aux affaires du territoire MRC. Au-delà du vote, l'élection du préfet est une occasion d'informer, de sensibiliser et de débattre des enjeux de ce palier territorial. Il y a donc lieu de croire qu'elle permet au public de s'approprier davantage ce territoire comme aire de gestion. Puisque l'élection est ponctuelle, nous supposons que la participation le sera aussi s'il n'y a pas d'ouverture sur le milieu par la suite (PÉ-expert). Cependant, la mise en place de mécanismes permanents permettra à ce levier de créer dynamisme et participation (PÉ-mobilisateur).

La dernière variable relève de la responsabilité du développement local dévolue en 2003. Bien qu’il soit difficile de mesurer pour l'instant l'impact de cette mesure, on sait déjà qu'elle renferme un potentiel important pour devenir un lieu de synergie et de mobilisation des acteurs à l'échelle de la MRC. Le CLD étant composé d'élus et de personnes du milieu, il est déjà un organisme qui favorise une certaine participation. Cependant, dans la loi qui met en place les CLD, on donne beaucoup de latitude aux MRC pour élaborer des plans de développement pour le territoire. La gestion du développement local est obligatoire, mais la façon dont le Conseil MRC va l'appliquer peut prendre diverses formes. Ainsi, le développement local peut être délégué au CLD qui va gérer des fonds et des services d'aide aux entreprises sans concertation ni intégration avec d'autres organismes du milieu (CLD-gestion). Nous aurons alors un CLD de type gestionnaire. À l'opposé, les élus peuvent choisir une approche mobilisatrice (CLD-mobilisateur) pour que le développement local, par l'entremise du CLD, permette aux acteurs d'échanger de l'information, de travailler ensemble sur les enjeux et même d'élaborer une vision commune de développement. Cette façon de procéder vise davantage, selon nous, à faire en sorte que les territoires MRC soient des interfaces de développement.

\section{Au-delà du vote, l'élection du préfet est une occasion d'informer, de sensibiliser et de débattre des enjeux de ce palier territorial.}

\section{Le dynamisme des MRC}

Le schéma 2 propose des scénarios en fonction des axes du dynamisme des MRC et de l'appropriation des leviers (prise en main des leviers, outils, mesures, etc.). Les Conseils MRC et le secteur public contribuent, par la mise en place d'organisations dans diverses fonctions (culture, loisirs, environnement, santé, éducation, etc.), à favoriser une appropriation de ce territoire comme aire de gestion. Les interventions de l'État ont aussi des effets sur le nombre de responsabilités qui relèvent de ce niveau territorial. Par exemple, le développement local est devenu une compétence obligatoire pour les MRC en 2003 à la suite d'une réglementation. Elles doivent élaborer une vision stratégique du développement économique, social, culturel et environnemental (MAMR). Nous considérons que les compétences obligatoires (CO), facultatives (CF) et les autres outils, notamment l'élection du préfet (PÉ) et le développement local (CLD), sont autant de leviers qui peuvent faire l'objet d'une appropriation plus ou moins forte selon l'approche.

Les MRC ont des compétences obligatoires et facultatives. L'appropriation sera abordée de façon différente puisque dans un cas il y a obligation et dans l'autre non. Dans le premier cas, nous mesurons le niveau d'appropriation et le dynamisme en fonction de l'approche utilisée par le Conseil MRC (technocratique ou participationniste). Prenons une compétence obligatoire comme l'aménagement pour illustrer notre propos. Le schéma d'aménagement peut être confectionné par un expert embauché à l'externe (COexpert). Le conseil peut mandater un expert et faire une consultation restreinte (CO-ponctuelle) au sein des principaux décideurs. Il peut aussi effectuer une 
consultation élargie auprès de plusieurs organismes (CO-intégration). Nous avons là trois exemples différents qui illustrent les possibilités offertes au Conseil MRC pour l'appropriation d'une compétence obligatoire (schéma 2). Le cas des compétences facultatives (CF) est différent puisque le Conseil MRC est libre de les exercer ou non (adoption d'un plan de développement, logement social, fonds d'investissement locaux, etc.). Le premier niveau d'appropriation se définit alors par la décision d'exercer ou non ces compétences, et le second par l'approche utilisée. Nous considérons ici que l'ajout de nouvelles compétences au palier MRC est une appropriation forte. Soulignons aussi qu'il est possible, pour des municipalités, de déléguer des responsabilités vers le territoire MRC.

\section{Schéma 2 - Dynamisme des MRC}

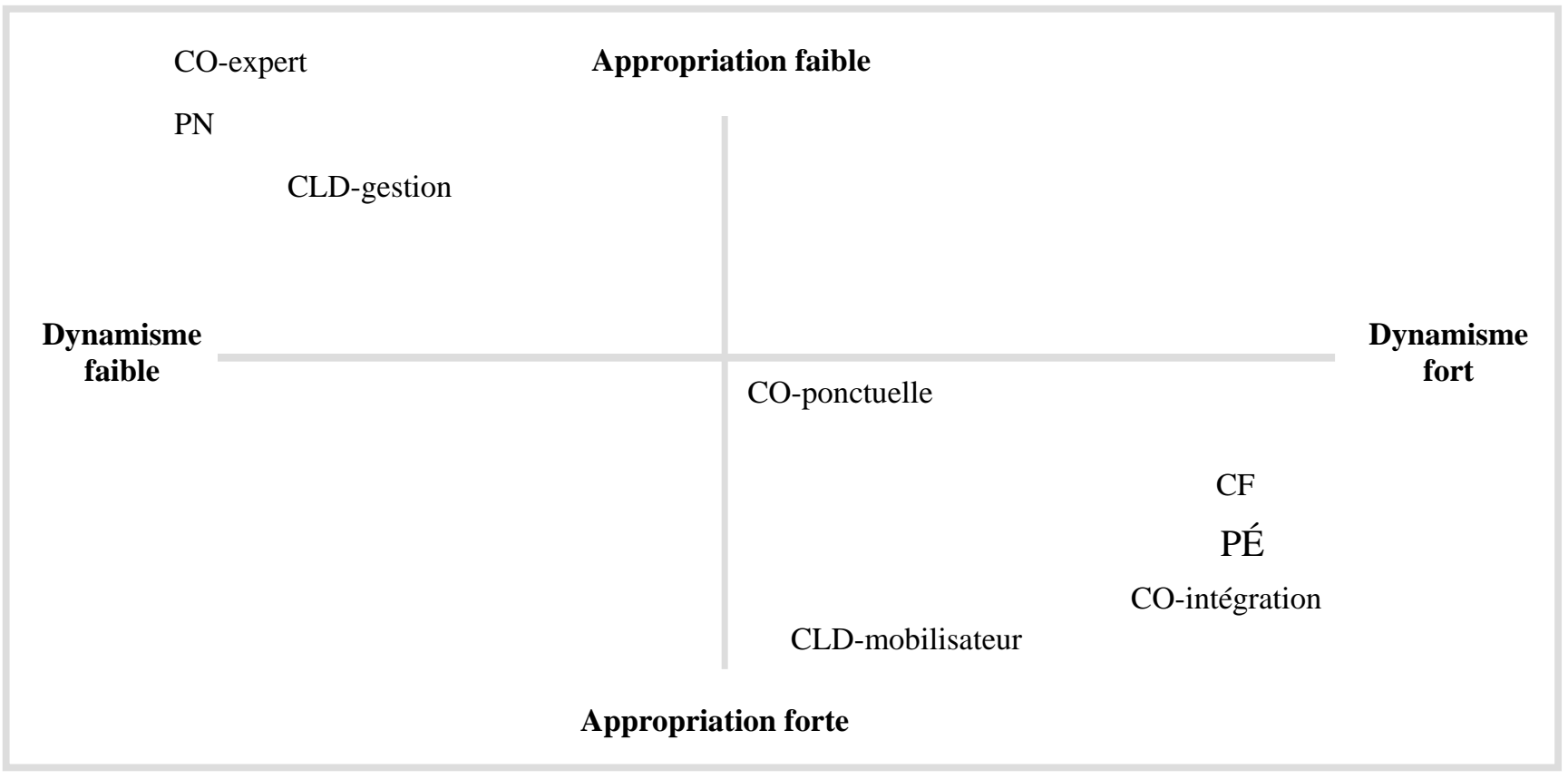

L'élection du préfet au suffrage universel est un outil potentiel d'appropriation. Il y a actuellement sept MRC qui ont un préfet élu et une seule a fait une demande pour l'année 2005. Malgré les incitatifs proposés par le ministère des Affaires municipales et des Régions et les nombreux avantages qu'offre cet outil ${ }^{6}$, très peu de Conseils MRC misent sur ce levier. L'augmentation du leadership, la neutralité du préfet, la crédibilité, la capacité de faire de l'arbitrage (ruralurbain, local-régional) et la visibilité auprès de la population sont des avantages importants cités par les préfets élus. Nous croyons qu'il s'agit d'un outil qui n'est pas négligeable et qui ouvre la voie à deux autres scénarios pour les MRC qui ont le choix de nommer le préfet (PN) ou d'utiliser le suffrage universel (PÉ). Dans ce cas, il n'y a pas d'autres options. Finalement, comme nous l'avons exprimé précédemment, le développement local, par l'entremise des Centres locaux de développement, offre des scénarios d'appropriation. L'appropriation plus faible consiste à lui donner une approche de gestion (CLD-gestion), alors que celle qui consiste à mobiliser le milieu relève de l'appropriation forte du levier (CLD-mobilisateur).

\section{Conclusion}

Notre réflexion a permis de mettre en évidence certains scénarios qui auront des impacts sur la place qu'occuperont les territoires MRC dans l'avenir. Depuis leur implantation, les MRC se sont construites par la conjugaison des forces endogènes (volonté et dynamisme du milieu) et exogènes (interventions de l'État et des organisations nationales). Celles-ci sont encore présentes. Cependant, nous constatons que certains leviers existent et que l'intensité de leur appropriation génère des scénarios différents pour l'avenir de ce territoire. À cet effet, nous pouvons citer le préfet à temps plein, le préfet élu et l'exercice de fonctions facultatives. Outre l'utilisation des outils, 
nous supposons que le type d'intervention utilisé par le Conseil MRC ouvre la voie à d'autres scénarios. La participation du milieu et la concertation sont des facteurs de développement reconnus depuis fort longtemps. Cette approche est peu coûteuse et permettrait une utilisation accrue de ce territoire.

\section{Nous constatons que certains leviers existent et que l'intensité de leur appropriation génère des scénarios différents pour l'avenir de ce territoire.}

Dans le continuum de possibilités qui s'offrent aux MRC, nous pouvons toutefois dégager de grandes directions d'avenir. Les Conseils MRC peuvent, d'une part, renforcer le territoire en adoptant une approche ouverte sur le milieu, que ce soit par la consultation, l'éclatement des fonctions dans de petits comités, une gestion des CLD démocratique et mobilisatrice, etc. ou, d'autre part, exercer les fonctions en utilisant l'expertise interne, et externe, selon les besoins. Dans sa volonté de renforcer le territoire MRC, l'État québécois peut offrir diverses mesures et laisser les milieux s'approprier les outils en fonction de leurs besoins et de leurs réalités. Le gouvernement peut aussi, par décret, imposer certaines mesures comme il l'a déjà fait par le passé. Notre réflexion nous porte à croire que les territoires MRC peuvent choisir de se renforcer comme aire de gestion ou d'explorer davantage la voie de la participation et de l'intégration pour devenir une véritable aire de développement.

\section{Notes et références}

1 Fortin, G. et L. Parent L. (1983), Les MRC et leur capacité d'extension, Montréal, INRS-urbanisation.

2 Proulx, M.-U. (1992), Le secteur public territorial : MRC 1992, Chicoutimi, Université du Québec à Chicoutimi.

3 Proulx, J. (2001), « La dynamique organisationnelle des territoires de MRC », Cahiers de géographie du Québec, vol. $45, n^{\circ} 124$, avril.

4 Proulx, M.-U. (1992), op. cit.

5 Jean, N. (1999), Les territoires MRC du Québec : deux décennies d'évolution dans la diversité, Chicoutimi, Université du Québec à Chicoutimi.

6 Selon une enquête faite auprès des MRC en 2005. 